\title{
Autonomous planning and control of soft untethered grippers in unstructured environments
}

\author{
Federico Ongaro $^{1}$ (D) . Stefano Scheggi ${ }^{1}$. ChangKyu Yoon $^{2} \cdot$ Frank van den Brink $^{1}$. \\ Seung Hyun $\mathrm{Oh}^{2}$ • David H. Gracias ${ }^{2}$ Sarthak Misra ${ }^{1,3}$
}

Received: 14 June 2016 / Revised: 14 August 2016 / Accepted: 25 August 2016 / Published online: 12 September 2016

(C) The Author(s) 2016. This article is published with open access at Springerlink.com

\begin{abstract}
The use of small, maneuverable, untethered and reconfigurable robots could provide numerous advantages in various micromanipulation tasks. Examples include microassembly, pick-and-place of fragile micro-objects for lab-on-a-chip applications, assisted hatching for in-vitro fertilization and minimally invasive surgery. This study assesses the potential of soft untethered magnetic grippers as alternatives or complements to conventional tethered or rigid micromanipulators. We demonstrate closed-loop control of untethered grippers and automated pick-and-place of biological material on porcine tissue in an unstructured environment. We also demonstrate the ability of the soft grippers to recognize and sort non-biological micro-scale objects. The fully autonomous nature of the experiments is made possible by the integration of planning and decision-making algorithms, as well as by closed-loop temperature and electromagnetic motion control. The grippers are capable of completing pick-and-place tasks of biological material at an average velocity of $1.8 \pm 0.71 \mathrm{~mm} / \mathrm{s}$ and a drop-off error of $0.62 \pm 0.22 \mathrm{~mm}$. Color-sensitive sorting of three microscale objects is completed at a velocity of $1.21 \pm 0.68 \mathrm{~mm} / \mathrm{s}$
\end{abstract}

F. Ongaro, S. Scheggi and C. Yoon contributed equally to this work.

Federico Ongaro

f.ongaro@utwente.nl

1 Surgical Robotics Laboratory, University of Twente, Enschede, 7522 NB, The Netherlands

2 The Johns Hopkins University, Baltimore, MD 21218, USA

3 University of Groningen and University Medical Centre Groningen, Groningen, 9713 GZ, The Netherlands and a drop-off error of $0.85 \pm 0.41 \mathrm{~mm}$. Our findings suggest that improved autonomous untethered grippers could augment the capabilities of current soft-robotic instruments especially in advancedtasks involving manipulation.

Keywords Soft robotics · Gripper · Self-folding · Biocompatible $\cdot$ Autonomous

\section{Introduction}

Small-scale robots have shown promising results in a broad variety of tasks, ranging from micromanipulation [1-4] and microassembly $[5,6]$ to minimally invasive surgical (MIS) interventions, such as biopsies [7] and targeted drug delivery [8-11]. In many of these tasks there is a need to move and transport objects with high precision in unstructured environments. Examples include pick-and-place of fragile objects for lab-on-a-chip applications, assisted hatching for in-vitro fertilization, assembly and repair of printed circuit boards (PCB) and of microelectromechanical systems (MEMS), angioplasty and embolization [12-16]. Untethered small-scale robots with soft, maneuverable and reconfigurable components could perform these procedures, improving functionality. For this purpose, the robots would have to possess reliable and robust motion and grasping capabilities. Soft grippers might represent a viable solution. The reliability and robustness of large-scale soft grippers have been heavily demonstrated, as they are a reality in the market for manipulators [17-19]. Additionally, closed-loop control would be necessary to achieve the required high levels of precision and robustness.

However, the implementation of closed-loop control at such scales presents significant challenges. Furthermore, small-scale robots have to satisfy at least four requirements 
for applicability. They must: (1) possess an untethered structure that allows them to move in tortuous environments; (2) allow manipulation along accurate trajectories in unstructured environments; (3) have sizes that allow them to reach and move within constrained environments; and (4) be capable of detecting and promptly reacting to variations of their surroundings. Various untethered agents capable of manipulation on small size scales have been described previously [20-23]. Among these, self-folding hydrogel soft grippers allow the decoupled control of motion and grasp [24]. Their ability to wirelessly harness power from the surrounding environment offers a solution to requirement \#(1) [7, 25]. They can be fabricated in sizes that are compatible with applications such as in-vitro fertilization, PCB and MEMS assembly, and allow access to the major vascular vessels, satisfying requirement \#(3) [26-28]. Furthermore, requirements \#(2) and \#(4) can be fulfilled by using accurate decision-making and tracking algorithms, closed-loop control, and fast online trajectory planning that exploits the ferromagnetic properties of the grippers. The synergy of such algorithms can make the system fully-autonomous, greatly increasing performance consistency and success rates [29].

Although several pathfinding algorithms have been developed in recent years, only few have been applied to magnetic agents in an experimental scenario. Khalil et al. implemented a 2D path planner based on the A* algorithm and the Artificial Potential Field approach for paramagnetic microparticles [30]. A path planner based on the Rapidly-exploring Random Tree algorithm was developed for biological cell transportation using optical tweezers in [31]. Recently, an experimental comparison of six path planning algorithms when applied to the motion control of paramagnetic microparticles was presented in [32].

In this work, we show the potential of soft, untethered grippers in tasks that involve autonomous manipulation, as well as obstacle recognition and manipulation in unstructured environments (Fig. 1). Two experiments are performed for this purpose. In the first, soft bilayer grippers are used to perform the pick-and-place of soft irregularly-shaped biological material in the presence of both static and dynamic obstacles. The second experiment demonstrates that soft grippers can autonomously recognize, classify, and manipulate regularly-shaped micro-scale rigid objects. In both experiments, the soft grippers utilize their six tips to grasp a micro-scale object and constrain it in all directions. (Fig. 1).

It should be noted that soft-grippers were previously manipulated using robotic control [22]. However, there are several important differences between our work and this previously reported study. Firstly, previously utilized grippers had a homogeneous mechanical composition, whereas our soft grippers are designed with rigid segments and flexible joints. This design feature seeks to mimic biological

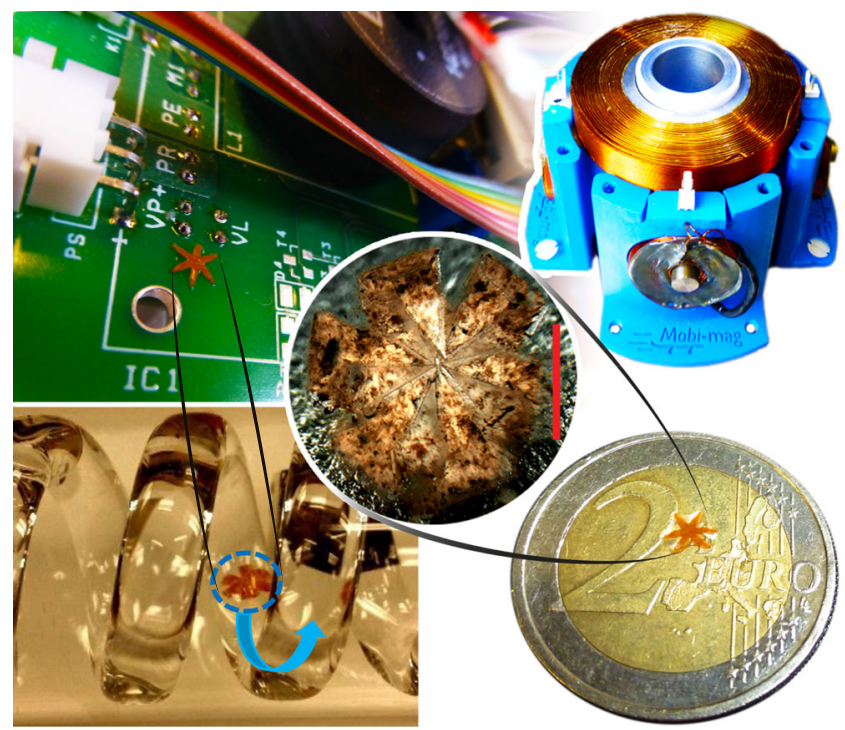

Fig. 1 Top Left: Image of a soft untethered gripper on a printed circuit board. Top Right: The electromagnetic setup consisting of six orthogonally-oriented electromagnetic coils. The electromagnetic coils have a cutoff frequency of $70 \mathrm{~Hz}$ and a maximum current rating of $1 \mathrm{~A}$. The system generates a maximum magnetic field and magnetic field gradient of $15 \mathrm{mT}$ and $60 \mathrm{mT} / \mathrm{m}$, respectively. Center: Image of the closed gripper. Bottom Left: Video snapshot images of the gripper navigating through a tube with an inner diameter of $3.2 \mathrm{~mm}$, a size smaller than most of the major vessels in the human body. Bottom Right: Image of the gripper on a $2 €$ coin. The scale bar is $0.4 \mathrm{~mm}$

appendages such as hands, and ensures a robust grasp of the transported microscale objects, even in the case of abrupt jerks. Secondly, as compared to previous work in which the gripper itself was not magnetic but rather was moved by a magnetic object, we have succeeded in doping the grippers themselves with magnetic nanoparticles to allow motion control even when the grippers are not grasping a magnetic object. Furthermore, bulk magnetic doping was chosen instead of a surface coating to preserve the soft material characteristics of the gripper and prevent the corrosion or delamination of the magnetic cladding in media such as acid fluids or blood. Additionally, in this work the grippers are fully autonomous; i.e., they are able to interact with the environment and automatically adapt their behavior in real-time to changes in the scenario. Consequently, the aforementioned tasks can be autonomously performed in a faster, safer, and more robust way. Finally, a novel high sampling rate technique that makes use of an XYZ Cartesian robot is used in order to model and validate the electromagnetic system. Such validation at high density and resolution not only ensures the reliability of the model, but also enables the reliable computation of the magnetic field gradient.

The rest of the paper is organized as follows. Section 2 describes the fabrication of the soft hydrogel grippers. The techniques used to track and detect the grippers are 


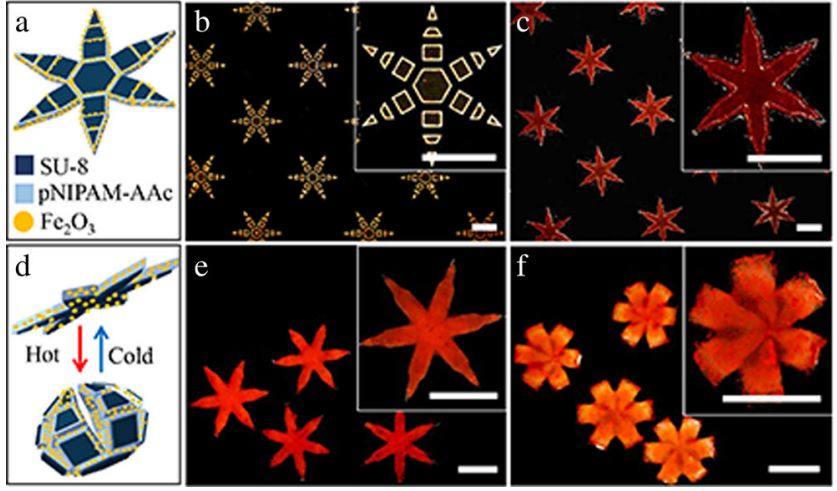

Fig. 2 Schematic and experimental images of the fabrication and operation of the soft bilayer grippers. a Schematic showing the bilayer composed of a stiff, segmented, SU-8 polymer coupled with a $5 \%(\mathrm{w} / \mathrm{w})$ magnetic nanoparticle $\left(\mathrm{Fe}_{2} \mathrm{O}_{3}\right)$ doped thermally stimuli responsive poly(N-isopropylacrylamide-co-acrylic acid) (pNIPAMAAc) layer (b-c). Experimental images after wafer scale patterning of the two layers. d Schematic and (e-f) experimental images showing operation of the grippers with reconfiguration from, $\mathbf{e}$ a flat state at room temperature to, $\mathbf{f}$ a closed state in which the temperature is raised to $25.3^{\circ} \mathrm{C}$

reviewed in Section 3. Section 4 presents the motion planner used to move them along obstacle-free paths, here also the modeling of the electromagnetic setup and its validation are described. The experimental validation is presented in Section 5. Finally, Section 6 summarizes the main contributions of this paper, and proposes possible avenues for future research.

\section{Fabrication of the hydrogel grippers}

The detailed gripper fabrication process has been described previously [28] with one design change in that we replaced the stiff polymer with SU-8, a widely available photopatternable polymer. Briefly, bilayer grippers (Fig. 2a) were composed of lithographically photopatterned segmented SU-8 (Fig. 2b) and continuous poly (N-isopropylacrylamide-co-acrylic acid) (pNIPAM-AAc) (Fig. 2c). Furthermore, in order to make magnetically responsive soft grippers, $5 \%(\mathrm{w} / \mathrm{w})$ biocompatible iron (III) oxide $\left(\mathrm{Fe}_{2} \mathrm{O}_{3}\right.$, Sigma-Aldrich, St. Louis, USA) $50 \mathrm{~nm}$ nanoparticle powder was mixed with the pNIPAM-AAc solution prior to crosslinking. The soft grippers open and close reversibly due to a phase transition and associated swelling or shrinkage in the PNIPAM-AAc layer in response to temperature changes (Fig. 2d-f). When fully opened, the grippers have an hexagram shape with a tip-to-tip distance of $4 \mathrm{~mm}$. When fully closed, the grippers have the shape of a sphere with a $0.4 \mathrm{~mm}$ radius.

The $\mathrm{F}_{2} \mathrm{O}_{3}$ nanoparticle patterning and the magnetic field gradient generated by an array of six orthogonal iron-core electromagnets (Fig. 1) can be used for closed-loop motion control of the grippers. To perform autonomous, accurate and robust pick-and-place of biological material in unstructured environments, the following modules are necessary: (1) an accurate image-guided tracking algorithm; (2) a reactive motion planner which uses the information from the tracker to compute suitable motion control inputs for the gripper. In the next section, we describe the tracking algorithm used to estimate the pose of the gripper.

\section{Detection and tracking of the grippers}

Let $\mathbf{p}=\left[\begin{array}{ll}x, & y\end{array}\right]^{T} \in \mathbb{R}^{2 \times 1}$ be the position of a gripper in $2 \mathrm{D}$ space. The state of the gripper is defined as $\mathbf{x}=\left[x, y, v_{x}, v_{y}\right]^{T} \in \mathbb{R}^{4 \times 1}$ where $v_{x}, v_{y}$ represent its velocities. Let us consider the miniaturized gripper as a second order system controlled by applying suitable force inputs.

The tracking algorithm is shown in Fig. 3 and estimates the state $\hat{\mathbf{x}}$ and the configuration (open/close) of the

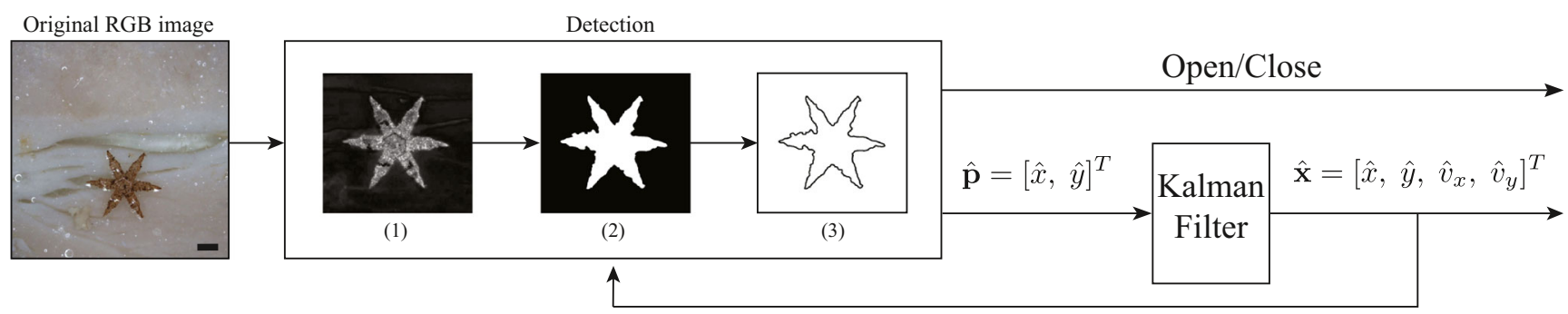

Fig. 3 Flowchart showing the tracking process: From left to right, a Red-Green-Blue (RGB) image is taken from the microscope. In the detection phase, the image is converted to Hue-Saturation-Value (HSV) color space. (1) Bounds on the coordinates of the selected color space are employed in order to accurately detect the gripper. (2) Adaptive threshold, morphological filtering, and opening are used. Then, the adjacent blobs are merged and kept for further consideration. (3) A
Discrete Fourier Transform is applied on the contour of the segmented area in order to detect the pose $\hat{\mathbf{p}}=\left[\begin{array}{ll}\hat{x}, & \hat{y}\end{array}\right]^{T}$ and the configuration (open/close) of the gripper. Finally, a Kalman filter is used to estimate the state $\hat{\mathbf{x}}$ of the gripper, where $\hat{v}_{x}, \hat{v}_{y}$ represent the estimated velocities of the agent. The estimated state of the gripper is used in the next frame to speed up the detection procedure. The scale bar is $1 \mathrm{~mm}$ 
gripper at runtime. In the initial phase, it determines the position $\hat{\mathbf{p}}$ of the gripper. This phase is composed of two steps. The first step extracts the contour of the gripper from the binarized color-saturation level of the image. The input to the proposed method is a $1024 \times 1024$ Red-GreenBlue (RGB) color image acquired using a Charge-Coupled Device (CCD)connected to a microscopic lens. In the presented work, the Hue-Saturation-Value (HSV) color space is selected. Then, the coordinates of the selected color space are bounded; i.e., specific values of the axis of the color space are selected in order to detect a particular color. The bounds are chosen empirically; i.e., by examining the distribution of colors in a preselected set of images. Morphological filtering and opening are used in order to segment the image. Finally, small blobs are removed and the remaining, adjacent blobs are merged and kept for further consideration.

In the second step, normalized Fourier Descriptors (FDs) $Z_{k}, k \in[-15, \ldots, 16]$ are obtained by applying the one-dimensional Discrete Fourier Transform (DFT) to the complex-valued representation of the $2 \mathrm{D}$ contour points. FDs provide us with the position and scale of the gripper [33]. To increase noise rejection, we ignore all the contours that are not 6 -fold symmetric by only tracking objects that satisfy the following conditions:

$Z_{-5}>\frac{2}{N-2}\left(\sum_{k=-15}^{16} Z_{k}-Z_{1}\right), Z_{7}>\frac{2}{N-2}\left(\sum_{k=-15}^{16} Z_{k}-Z_{1}\right)$.

Furthermore, we set a threshold constraint on the ratio between the number of pixels of the perimeter and of the area of the contour. This value must be between 0.5 and 1 in order to avoid erroneous tracking of other 6-fold symmetric objects.

To compute the estimated state $\hat{\mathbf{x}}$ from position measurements $\hat{\mathbf{p}}$, we implement a standard Kalman filter [34] to consider the second order model of the gripper and control-inputs. Assuming a constant sampling time $\Delta t$ of the system, the Kalman filter provides an estimation of the current state $\hat{\mathbf{x}}$ as well as a one-step ahead prediction of $\hat{\mathbf{x}}$. The process and measurement noises are obtained from zero mean multivariate Gaussian distributions $N(0, \mathbf{Q})$ and $N(0, \mathbf{R})$, respectively, where $\mathbf{Q} \in \mathbb{R}^{4 \times 4}$ is the process noise covariance matrix and $\mathbf{R} \in \mathbb{R}^{2 \times 2}$ is the measurement noise covariance matrix. $\mathbf{Q}$ is determined by empirically tuning its parameters, while $\mathbf{R}$ is computed by analyzing offline the tracked positions of the gripper using a zero-phase filter.

To speed up the detection procedure, temporal continuity is exploited to track the grippers in a sequence of frames. Given the estimated state $\hat{\mathbf{x}}$ of the tracked gripper from the previous frame, the image pixels that are within a preset range from that estimation are kept, whereas the remaining pixels are discarded. The proposed tracker runs at an average frame rate of 50 frame per second on a PC that has an Intel Xeon CPU $3.2 \mathrm{GHz}$ processor and $8 \mathrm{~GB}$ of RAM.

\section{Motion planning and control}

The estimated state $\hat{\mathbf{x}}$ of the gripper is then provided to a motion planner for the computation of a collision-free trajectory. Among possible motion planners, a Rapidlyexploring Random Tree (RRT) is used. The RRT outputs forces that are mapped to currents at the electromagnets. For this purpose, a force-current map is developed using Finite Element Model (FEM) analysis.

\subsection{Motion planning}

The RRT is able to deal with real-valued spaces of extremely high dimension while handling the dynamics of the system. The planner is rooted at the gripper's initial state $\mathbf{x}_{\text {start }}=$ $\hat{\mathbf{x}}$. At each iteration, the algorithm samples a collision-free state $\mathbf{x}_{\text {sample }}$ in the environment, finds its nearest neighbors $\mathbf{x}_{n n}$ in the tree, and computes feasible control forces $(\mathbf{F} \in$ $\mathbb{R}^{3 \times 1}$ ) that grow $\mathbf{x}_{n n}$ toward $\mathbf{x}_{\text {sample }}$. The output of the RRT is a motion plan $\Gamma$;

$\Gamma=\left[\left(\mathbf{x}_{\text {start }}, \mathbf{F}_{\text {start }}\right), \ldots,\left(\mathbf{x}_{S}, \mathbf{F}_{S}\right)\right]$,

where $S$ is the number of steps (Algorithm 1). Each entry of the motion plan $\Gamma$ represents a control input that is applied to the agent after every time interval $\Delta t$. Unlike path planning algorithms, which provide waypoints to the robot, RRTs directly provide feasible control inputs $\mathbf{F}$.

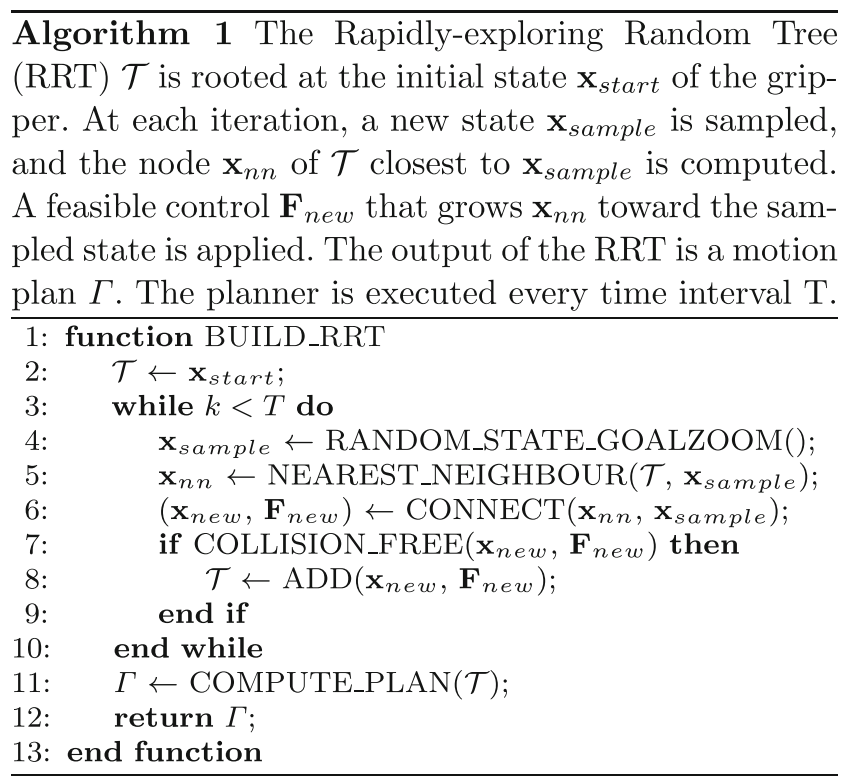


Table 1 Quality indices for the $\mathrm{x}-\left(\mathrm{B}_{x}\right)$ and $\mathrm{y}-\left(\mathrm{B}_{y}\right)$ components of the magnetic field for the obtained fitting function

\begin{tabular}{lllll}
\hline & $\operatorname{SSE}\left(\mathrm{T}^{2}\right)$ & $R^{2}$ & $\bar{R}^{2}$ & $\mathrm{RMSE}(\mathrm{T})$ \\
\hline $\mathrm{B}_{x}$ & $4.7 \times 10^{-7}$ & 1.0000 & 1.000 & $6.7 \times 10^{-6}$ \\
$\mathrm{~B}_{y}$ & $2.9 \times 10^{-7}$ & 1.0000 & 1.000 & $5.3 \times 10^{-6}$ \\
\hline
\end{tabular}

The table reports Sum Squared Error (SSE), R-square $\left(R^{2}\right)$, Adjusted R-square $\left(\bar{R}^{2}\right)$, and Root Mean Square Error (RMSE)

In the experimental validation, an RRT-GoalZoom policy is used to generate a new random state based on a biased coin toss [35]. The algorithm relies on this state to choose a random sample from either a region around the goal or from the whole space. During the planning procedure, instead of extending the RRT by an incremental step, the algorithm iterates until the random state or an obstacle is reached. Finally, a fixed planning time interval of $T=0.1 \mathrm{~s}$ is selected. At the beginning of the task, the RRT computes the motion plan. Successively for each period of duration $T$, the system updates the planned trajectory that will be ready for the next planning time interval $T$. The proposed motion planner allows the system to run autonomously without any need for human intervention during standard operations. This not only reduces the system response time below the average human reaction time (400 ms [36]) to an average of $100 \mathrm{~ms}$, but also lessens if not eliminates - the need for human supervision. This, combined with the higher repeatability of fully-autonomous systems, greatly increases performance consistency and success rates.

\subsection{FEM modeling and validation}

Once the RRT planner has computed the obstacle-free motion plan $\Gamma$, suitable currents have to be applied to the magnetic coils in order to exert the desired forces $\mathbf{F}_{i}, i=1, \ldots, S$ on the agent. For this purpose, we use FEM analysis (COMSOL Multiphysics, COMSOL, Stockholm, Sweden) to develop a map between the current at the electromagnets and the magnetic flux density in the workspace. The FEM analysis is successively compared with the measurements of a 3-axis 3MH3 Teslameter (SENIS AG, Baar, Switzerland). To obtain high-density measurements an XYZ Cartesian robot moves the probe through the entire workspace at a velocity of $0.5 \mathrm{~mm} / \mathrm{s}[37$, 38]. This technique allows us to compare the results at a
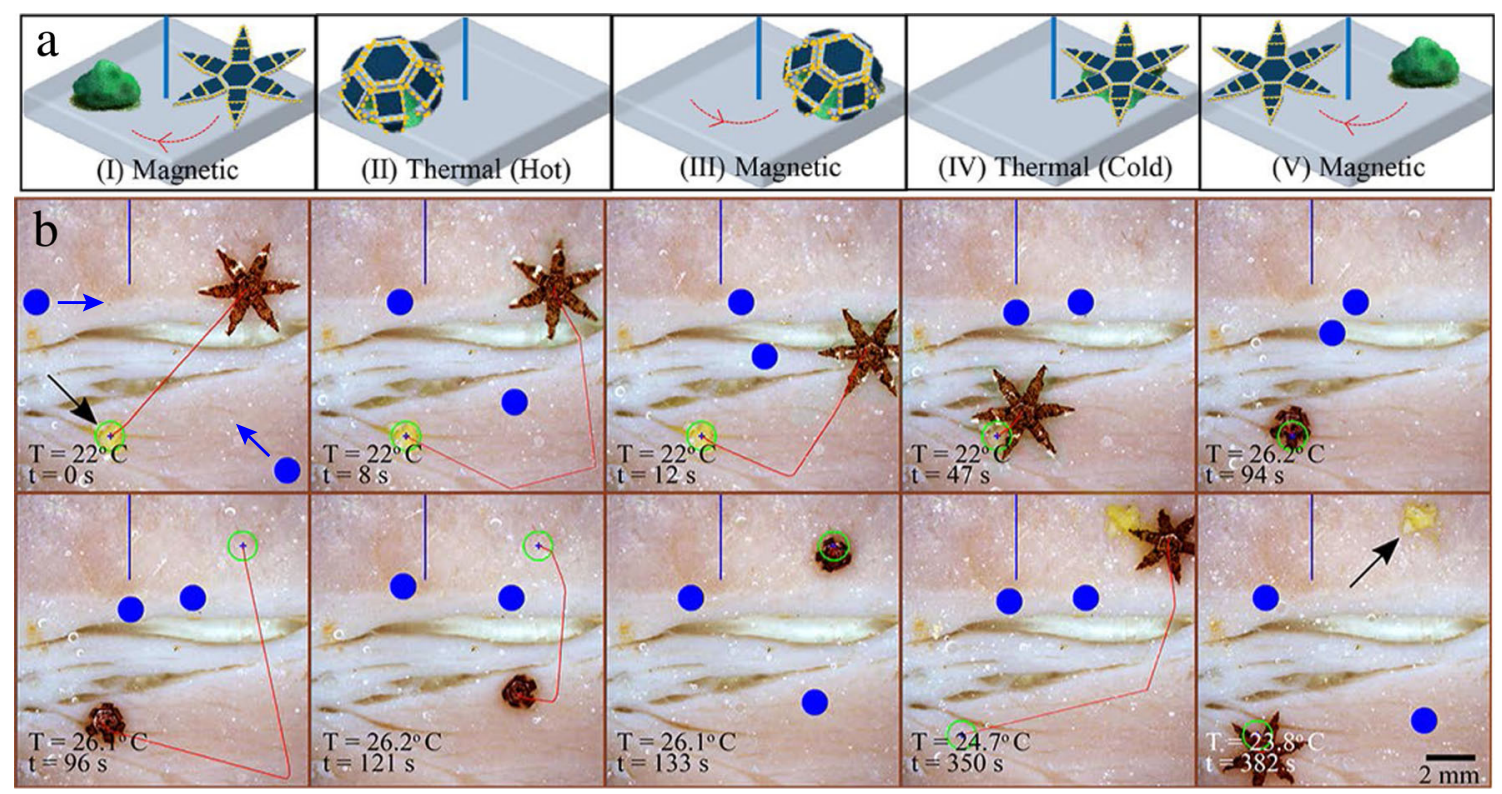

Fig. 4 a Schematic of a representative obstacle avoidance and biological manipulation experiment. b Video snapshots of the gripper during pick-and-place of biological material in an unstructured environment on porcine tissue. The virtual obstacles are shown in blue; the circular ones are dynamic, while the wall on top of the workspace is static. The blue arrows represent the initial motion directions of the dynamic obstacles. The velocity of the horizontally and diagonally moving obstacle is $0.35 \mathrm{~mm} / \mathrm{s}$ and $0.5 \mathrm{~mm} / \mathrm{s}$, respectively. The targeted position is represented the blue cross inside the green circle. The planned obstacle-free trajectory to reach the reference is represented as a red line. The gripper autonomously detects the yolk while a Rapidlyexploring Random Tree motion planner computes, and continuously updates, an obstacle-free trajectory in order to reach it. Once the target is reached, the temperature is increased until the system classifies the gripper as closed. An obstacle-free trajectory then guides the gripper to the target where the temperature is decreased until the gripper is marked as open. Finally, the gripper is moved away from the drop-off area. Times ( $\mathrm{t}$ ) and temperatures $(\mathrm{T})$ are shown on the bottom-left corner of each snapshot. Please refer to the accompanying video for this experiment 
resolution of $6.8 \times 10^{6}$ dots $/ \mathrm{m}^{2}$ using a total of 1523 samples in the $1.2 \times 1.2 \mathrm{~cm}$ workspace. The average mismatch and its standard deviation between FEM and measurements - when the electromagnets are fed the maximum current of $1 \mathrm{~A}-$ are $7.4 \times 10^{-3} \pm 2.2 \mathrm{mT}$ and $0.073 \pm 0.045$ radians for field magnitude and orientation, respectively. The $x-$ and $y$-components of the field are then fitted to a quadratic Lowess (LOcally WEighted Scatter plot Smooth) function using MATLAB (MathWorks Inc., Natick, United States). The quality indices of such functions are reported in Table 1. The quadratic Lowess functions are then used to compute the force-current map as described in $[39,40]$.

\subsection{Temperature control}

The configuration control is provided by a Peltier element. This element controls the temperature of the environment and, consequently, the configuration (open/close) of the gripper. The configuration, determined using FD, is used as feedback for the closed-loop grasp control. In the next section, an experimental validation is performed in order to demonstrate the ability of the grippers to manipulate both biological and non-biological materials in unstructured environments.

\section{Experimental validation}

An extensive evaluation is performed to assess the capabilities of the systems and demonstrate the ability of grippers to manipulate biological material in unstructured environments, and to detect and sort micro-scale objects. In the first experiment, one static and two dynamic virtual obstacles are avoided by the gripper that is at the same time performing pick-and-place of a piece of egg yolk (Fig. 4). The dynamic obstacles are two circles of $0.5 \mathrm{~mm}$ diameter, while the static obstacle is a wall with length of $4 \mathrm{~mm}$. The dynamic obstacles are modeled as first order linear holonomic systems. Their initial motion directions and speeds are reported in Fig. 4b. To simulate the tracking errors, we add zero-mean white Gaussian noise at a standard deviation $\sigma=0.5 \mathrm{~mm}$ to the $x-$ and $y$-coordinates of the dynamic obstacles. The noisy measured positions of the obstacles are provided to the motion planner in order to compute obstacle free paths. Please refer to the accompanying video showing
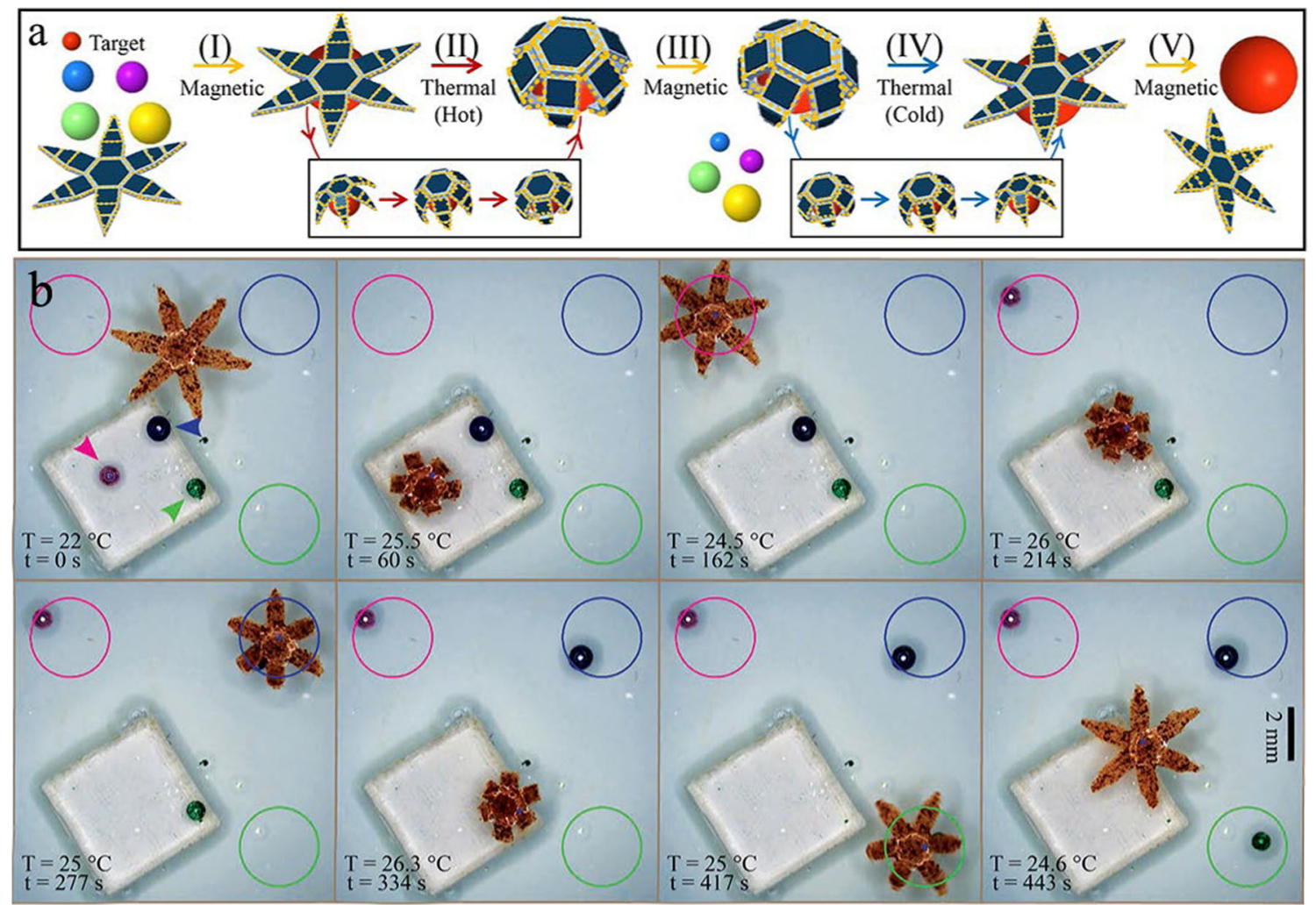

Fig. 5 a Schematic of a representative autonomous sorting experiment. b Video snapshots that show the gripper autonomously detecting and sorting differently colored beads, pick-and-placing them in the respectively colored drop areas. The gripper autonomously classifies the micro-scale objects, and estimates the configuration of the grippers from Fourier descriptors to determine if the desired configuration is reached. Times $(\mathrm{t})$ and temperatures $(\mathrm{T})$ are shown on the bottom-left corner of each snapshot. Please refer to the accompanying video for this experiment 
an example of the experiments. Egg yolk was chosen due to its high content of cholesterol and fat content (58\% of its dry weight), representative of materials that are often manipulated in MIS interventions. To increase the resemblance between the experimental scenario and operations in biological environments, the tasks are performed on a base of porcine tissue. Based on a total of five trials, this experiment is completed with an average error on the drop-off of the object of $0.62 \pm 0.22 \mathrm{~mm}$. The results were achieved at an average velocity of $1.81 \pm 0.71 \mathrm{~mm} / \mathrm{s}$ for loaded grippers and $2.08 \pm 0.39 \mathrm{~mm} / \mathrm{s}$ for unloaded grippers.

The second experiment tests if the grippers can autonomously recognize various objects and sort them. We perform an experiment in which the grippers recognize colored micro-scale objects and, one by one, pick-andplace them in the area marked with the corresponding color (Fig. 5). The manipulated objects are three spherical polyester beads of $0.5 \mathrm{~mm}$ diameter and $0.6 \pm 0.1 \mathrm{mg}$ weight. A color segmentation algorithm similar to that described in Section 3 is used to extract the position of the three objects. Please refer to the accompanying video showing an example of the experiments. A total of five trials are conducted with a velocity and error on the drop-off of the microscale object error of $1.21 \pm 0.68 \mathrm{~mm} / \mathrm{s}$ and $0.85 \pm 0.41 \mathrm{~mm}$ respectively. The average error of the micro-scale object always lays within the bounds of the radius of the drop-off location $(1.5 \mathrm{~mm})$.

\section{Conclusions}

The paper has demonstrated the capabilities of soft grippers to autonomously perform micromanipulation tasks. To further validate the proposed approach, we have performed tests on animal tissue in unstructured environments that contain time-varying obstacles. All the experiments were directed by the decision-making, path-planning, and closedloop control algorithms with no human intervention. Results show the ability of grippers to autonomously detect and adapt their behavior in response to changes in the surrounding environment. The demonstrated capabilities suggest that such grippers have the potential to enable delicate and precise manipulation in a broad variety of tasks, including microassembly, assisted fertilization and MIS. Moreover, due to their fully autonomous capabilities, they could find future applications in highly automatized processes as the population of PCB or the assembly of MEMS.

Future work will investigate the use of smaller scale grippers to offer more maneuverability and access to smaller environments. Also, 3D motion as well as operation in presence of fluid flow will be investigated. Operation in swarms will be studied to allow the parallelization of the micromanipulation tasks. Finally, we will exploit the metallic doped nature of these grippers in order to investigate the use of ultrasound or other clinically compatible imaging systems [41, 42].

Acknowledgments This project (ROBOTAR) has received funding from the European Research Council (ERC) under the European Union's Horizon 2020 Research and Innovation programme (Grant Agreement \#638428). The research reported in this publication was also supported by the National Institute of Biomedical Imaging and Bioengineering of the National Institutes of Health (NIH) under Award Number R01EB017742. The content is solely the responsibility of the authors and does not necessarily represent the official views of the NIH.

Open Access This article is distributed under the terms of the Creative Commons Attribution 4.0 International License (http:// creativecommons.org/licenses/by/4.0/), which permits unrestricted use, distribution, and reproduction in any medium, provided you give appropriate credit to the original author(s) and the source, provide a link to the Creative Commons license, and indicate if changes were made.

\section{References}

1. Wang J (2012) Cargo-towing synthetic nanomachines: towards active transport in microchip devices. Lab Chip 12(11):1944-1950

2. Pelrine R, Wong-Foy A, McCoy B, Holeman D, Mahoney R, Myers G, Herson J, Low T (2012) Diamagnetically levitated robots: An approach to massively parallel robotic systems with unusual motion properties. In: Proceedings of the IEEE International Conference on Robotics and Automation (ICRA), pp 739-744. IEEE

3. Miyashita S, Guitron S, Ludersdorfer M, Sung CR, Rus D (2015) Untethered miniature origami robot that self-folds, walks, swims, and degrades. In: Proceedings of the IEEE International Conference on Robotics and Automation, pp 1490-1496

4. Wong D, Steager EB, Kumar V (2016) Independent control of identical magnetic robots in a plane. IEEE Robotics and Automation Letters 1(1):554-561

5. Hoover AM, Fearing RS (2007) Rapidly prototyped orthotweezers for automated microassembly. In: Proceedings of the IEEE International Conference on Robotics and Automation (ICRA), pp 812-819. IEEE

6. Solovev AA, Xi W, Gracias DH, Harazim SM, Deneke C, Sanchez S, Schmidt OG (2012) Self-propelled nanotools. ACS Nano 6(2):1751-1756

7. Gultepe E, Randhawa JS, Kadam S, Yamanaka S, Selaru FM, Shin EJ, Kalloo AN, Gracias DH (2013) Biopsy with thermallyresponsive untethered microtools. Adv Mater 25(4):514-519

8. Martel S, Mathieu J-B, Felfoul O, Chanu A, Aboussouan E, Tamaz S, Pouponneau P, Yahia L, Beaudoin G, Soulez G et al (2007) Automatic navigation of an untethered device in the artery of a living animal using a conventional clinical magnetic resonance imaging system. Appl Phys Lett 90(11):114105

9. Arcese L, Fruchard M, Ferreira A (2012) Endovascular magnetically guided robots: navigation modeling and optimization. IEEE Trans Biomed Eng 59(4):977-987

10. Venugopalan PL, Sai R, Chandorkar Y, Basu B, Shivashankar S, Ghosh A (2014) Conformal cytocompatible ferrite coatings facilitate the realization of a nanovoyager in human blood. Nano letters 14(4):1968-1975 
11. Sitti M, Ceylan H, Hu W, Giltinan J, Turan M, Yim S, Diller E (2015) Biomedical applications of untethered mobile milli/microrobots. Proc IEEE 103(2):205-224

12. Cecil J, Powell D, Vasquez D (2007) Assembly and manipulation of micro devicesa state of the art survey. Robot Comput Integr Manuf 23(5):580-588

13. Gauthier M, Régnier S (2011) Robotic micro-assembly. Wiley

14. Chaillet N, Régnier S (2013) Microrobotics for micromanipulation. Wiley

15. Agnus J, Chaillet N, Clévy C, Dembélé S, Gauthier M, Haddab Y, Laurent G, Lutz P, Piat N, Rabenorosoa K et al (2013) Robotic microassembly and micrOmanipulation at femto-st. Journal of Micro-Bio Robotics 8(2):91-106

16. Chowdhury S, Jing W, Jaron P, Cappelleri DJ (2015) Path planning and control for autonomous navigation of single and multiple magnetic mobile microrobots, American Society of Mechanical Engineers

17. Amend JR Jr, Brown E, Rodenberg N, Jaeger HM, Lipson H (2012) A positive pressure universal gripper based on the jamming of granular material. IEEE Trans Robot 28(2):341-350

18. Majidi C (2014) Soft robotics: a perspective-current trends and prospects for the future. Soft Robotics 1(1):5-11

19. Softrobotics Inc. http://www.softroboticsinc.com, accessed: 201606-06

20. Tottori S, Zhang L, Qiu F, Krawczyk KK, Franco-Obregón A, Nelson BJ (2012) Magnetic helical micromachines: fabrication, controlled swimming, and cargo transport. Adv Mater 24(6):811816

21. Diller E, Sitti M (2014) Three-dimensional programmable assembly by untethered magnetic robotic micro-grippers. Adv Funct Mater 24(28):4397-4404

22. Fusco S, Sakar MS, Kennedy S, Peters C, Bottani R, Starsich F, Mao A, Sotiriou GA, Pané S., Pratsinis SE et al (2014) An integrated microrobotic platform for on-demand, targeted therapeutic interventions. Adv Mater 26(6):952-957

23. Kuo JC, Huang HW, Tung SW, Yang YJ (2014) A hydrogel-based intravascular microgripper manipulated using magnetic fields. Sensors Actuators A Phys 211:121-130

24. Ongaro F, Yoon C, van den Brink F, Abayazid M, Oh SH, Gracias DH, Misra S (2016) Control of untethered soft grippers for pick-and-place tasks. In: Proceedings of the IEEE/EMBS International Conference on Biomedical Robotics and Biomechatronics (BioRob), pp 299-304. IEEE

25. Gracias DH (2013) Stimuli responsive self-folding using thin polymer films. Current Opinion in Chemical Engineering 2(1):112-119

26. Hansen F, Mangell P, Sonesson B, Länne T. (1995) Diameter and compliance in the human common carotid artery-variations with age and sex. Ultrasound Med Biol 21(1):1-9

27. Malachowski K, Jamal M, Jin Q, Polat B, Morris CJ, Gracias DH (2014) Self-folding single cell grippers. Nano Lett 14(7):41644170
28. Breger JC, Yoon C, Xiao R, Kwag HR, Wang MO, Fisher JP, Nguyen TD, Gracias DH (2015) Self-folding thermo-magnetically responsive soft microgrippers. ACS Appl Mater Interfaces 7(5):3398-3405

29. Wang W, Liu X, Sun Y (2007) Autonomous zebrafish embryo injection using a microrobotic system. In: Proceedings of the IEEE International Conference on Automation Science and Engineering, pp 363-368

30. Khalil ISM, Keuning JD, Abelmann L, Misra S (2012) Wireless magnetic-based control of paramagnetic microparticles. In: Proceedings of the IEEE International Conference on Biomedical Robotics and Biomechatronics (BioRob), pp 460-466

31. Ju T, Liu S, Yang J, Sun D (2014) Rapidly exploring random tree algorithm-based path planning for robot-aided optical manipulation of biological cells. IEEE Trans Autom Sci Eng 11(3):649-657

32. Scheggi S, Misra S (2016) An experimental comparison of path planning techniques applied to micro-sized magnetic agents. In: Proceedings of the IEEE International Conference on Manipulation, Automation and Robotics at Small Scales (MARSS). In Press

33. Van der Heijden F (1994) Image based measurement systems: object recognition and parameter estimation

34. Gelb A (1974) Applied optimal estimation

35. LaValle SM, kuffner JJ (2000) Rapidly-exploring random trees: Progress and prospects. In: Algorithmic and Computational Robotics: New Directions, pp 293-308

36. Thorpe S, Fize D, Marlot C et al (1996) Speed of processing in the human visual system. Nature 381(6582):520-522

37. Vrooijink GJ, Abayazid M, Misra S (2013) Real-time threedimensional flexible needle tracking using two-dimensional ultrasound. In: Proceedings of the IEEE International Conference on Robotics and Automation (ICRA), pp 1688-1693

38. Vrooijink GJ, Abayazid M, Patil S, Alterovitz R, Misra S (2014) Needle path planning and steering in a three-dimensional nonstatic environment using two-dimensional ultrasound images. Int J Robot Res 33(10):1361-1374

39. Khalil ISM, Abelmann L, Misra S (2014) Magnetic-based motion control of paramagnetic microparticles with disturbance compensation. IEEE Trans Magn 50(10):1-10

40. Kummer MP, Abbott JJ, Kratochvil BE, Borer R, Sengul A, Nelson BJ (2010) Octomag: An electromagnetic system for 5-dof wireless micrOmanipulation. IEEE Trans Robot 26(6):1006-1017

41. Khalil ISM, Ferreira P, Eleutério R, de Korte CL, Misra S (2014) Magnetic-based closed-loop control of paramagnetic microparticles using ultrasound feedback. In: Proceedings of the IEEE International Conference on Robotics and Automation (ICRA), pp 3807-3812

42. Sánchez A, Magdanz V, Schmidt OG, Misra S (2014) Magnetic control of self-propelled microjets under ultrasound image guidance. In: Proceedings of the IEEE/EMBS International Conference on Biomedical Robotics and Biomechatronics (BioRob), pp 169-174 